\title{
NONLINEAR TRANSIENT ANALYSIS FOR LARGE-SCALE DYNAMICS OF MICROELECTROMECHANICAL SYSTEMS WITH THE MOR-PIM METHOD
}

\author{
Le Guan ${ }^{1}$, Jinkui Chu ${ }^{1 *}$, Jiali Gao ${ }^{2}$, Ran Zhang $^{2}$ \\ ${ }^{1}$ Key Laboratory for Dalian University of Technology Precision \& Non-traditional \\ Machining of Ministry of Education, 116023, P. R. China \\ chujk@dlut.edu.cn \\ ${ }^{2}$ Key Laboratory for Micro/Nano Technology and System of Liaoning Province, \\ Dalian, 116023, P. R. China
}

\begin{abstract}
Microelectromechanical Systems (MEMS) is difficult to take transient analysis due to the tight coupling between the multiple energy domains, typically nonlinear. An effective increment-dimensional precise integration method (PIM) combined with the model order reduction (MOR) technique based on Krylov subspace is present to solve large-scale nonlinear finite element dynamics systems. The numerical example of V-beam electro-thermal actuator is shown to demonstrate that the MORPIM method can achieve high precision and fast speed when solving the nonlinear dynamic equation with large-scale freedom.
\end{abstract}

Keywords- MEMS, Precise Integration Method, Model Order Reduction, Krylov

\section{INTRODUCTION}

The research field of MEMS has seen a rapid growth for the last two decades. During the micro and nano fabrication technology development, more and more micro/nano sensors, actuators and even system-on-chip with complexity structures and functions have been designed and taken into commercial application. The outstanding feature of MEMS is that it coupled multiple physical energy domains. Furthermore, if the systems contain the microelectronic devices for switching the non-electric signals to electric signals or some else signal processing units, the traditional finite element and boundary element tools are cumbersome and time consuming, i.e. are inappropriate to perform the transient analysis to overall system. Therefore, system-level modeling and simulation have become state of the art in MEMS design due to increasing demand for the computer aided engineering and design systems. The model order reduction technique has been extensively studied in recent years such as the superposition of basic function based on Galerkin method and the matrix subspace projection based on finite element equations [1]. The reduced order model is usually described by Ordinary Differential Equations (ODEs) or Differential Algebraic Equations (DAEs) in time. If all reduced order model of non-electric energy domains were build, then could be combined with the mature circuit model to take the system-level simulation for whole coupled micro-systems. Direct time-stepping methods have been the most popular and widely advocated in most commercial software for solving the system ODEs. In particular, the time difference/ $\theta$-difference method [2] plays a dominant role for a linear time-invariant system. With the direct iteration or Newton-Raphson iteration method, 
the finite difference method (FDM) can be extended to nonlinear analysis. However, the FDM usually brings errors and a number of numerical difficulties, such as stiffness of the problem. Zhong [3] proposed a very special explicit integration scheme, Precise Integration Method (PIM) for structural dynamic. The key component of the PIM is computing the matrix exponential. It can give precise numerical results almost equal to the exact solution at the integration point but it is more or less difficult due to the inverse matrix calculations and memory storage when solving the large-scale problem.

In this paper, we took the increment-dimensional precise integration method combined with the Krylov based approximation algorithm (called MOR-PIM) to solve large-scale nonlinear dynamics micro systems. The transient response result of finite element models for V-beam electro-thermal actuator was verified.

\section{THEORY BACKGROUND}

\subsection{An increment-dimensional precise integration method}

A general form of dynamics systems is given as

$$
\dot{\mathrm{v}}=\mathrm{A}(\mathrm{v}, \mathrm{t}) \mathrm{v}+\mathrm{f}
$$

where $\mathrm{v} \in \mathrm{R}^{\mathrm{n}}$ is the variable vector, $\mathrm{A}$ is $\mathrm{n} \times \mathrm{n}$ system matrix function, $\mathrm{f}$ is the external force vectors. It can also be written as

$$
\dot{\mathrm{v}}=\left(\mathrm{A}_{0}+\mathrm{A}_{1}(\mathrm{v}, \mathrm{t})\right) \mathrm{v}+\mathrm{f} \quad \text { or } \quad \dot{\mathrm{v}}=\mathrm{A}_{0} \mathrm{v}+\mathrm{f}_{\mathrm{c}}
$$

where $A_{0}$ is a time invariant matrix, and $A_{1}$ is time variant or is related to the unknown vector $v, f_{c}=f+A_{1}(v, t) v$ can be treated as some 'external force'. The existence of nonhomogeneous term $f_{c}$ has brought algorithmic complexity in PIM especially in largescale problems due to computing the inverse matrix. To eliminate the inverse matrix calculation, here a new state variable $\mathrm{v}_{\mathrm{n}+1} \equiv 1$ is introduced to equation (2) and the nonhomogeneous equations should be transformed into homogenous ones with dimensional expanding [4].

$$
\dot{\mathrm{V}}=\left[\begin{array}{c}
\dot{\mathrm{v}} \\
\dot{\mathrm{V}}_{\mathrm{n}+1}
\end{array}\right]=\left[\begin{array}{cc}
\mathrm{A}_{0} & \mathrm{f}_{\mathrm{c}} \\
0 & 0
\end{array}\right]\left[\begin{array}{c}
\mathrm{v} \\
\mathrm{v}_{\mathrm{n}+1}
\end{array}\right]=\mathrm{H}\left[\begin{array}{c}
\mathrm{v} \\
\mathrm{v}_{\mathrm{n}+1}
\end{array}\right]=\mathrm{HV}
$$

In the small time interval $t_{k}$ to $t_{k+1}, t_{k}=k \eta$, the matrix $H$ could regarded as invariant matrix. The homogeneous ODEs for initial value problems should be solved by PIM as follows: the general solution for equation can be given as

$$
\mathrm{V}=\exp (\mathrm{Ht}) \cdot \mathrm{V}_{0}
$$

$\mathrm{V}_{0}$ is initial value. The exponential matrix is described as Taylor expansion expression

$$
\exp (\mathrm{Ht})=\mathrm{I}_{\mathrm{n}}+\mathrm{Ht}+\frac{(\mathrm{Ht})^{2}}{2 !}+\frac{(\mathrm{Ht})^{3}}{3 !}+\frac{(\mathrm{Ht})^{4}}{4 !}+\cdots
$$

In a series of equal time step $\mathrm{t}_{0}=0, \mathrm{t}_{1}=\eta, \ldots \mathrm{t}_{\mathrm{k}}=\mathrm{k} \eta, \ldots$ for which 


$$
\mathrm{V}_{1}=\mathrm{V}(\eta)=\mathrm{TV}_{0}, \mathrm{~V}_{2}=\mathrm{V}(2 \eta)=\mathrm{TV}_{1}, \ldots, \mathrm{V}_{\mathrm{k}+1}=\mathrm{V}((\mathrm{k}+1) \eta)=\mathrm{TV}_{\mathrm{k}}, \ldots \quad \mathrm{T}=\exp (\mathrm{H} \eta)
$$

So the time step integration becomes the recurrence as equation (6). Therefore, the problem is reduced to commutating the exponential matrix $\mathrm{T}$. Let

$$
\mathrm{T}=\exp (\mathrm{H} \eta) \equiv[\exp (\mathrm{H} \eta / \mathrm{m})]^{m}
$$

where $\mathrm{m}$ is an arbitrary integer. It is suggested to select

$$
\mathrm{m}=2^{\mathrm{N}} \text { such as } \mathrm{N}=20, \mathrm{~m}=1048576
$$

$\tau=\eta / \mathrm{m}$ is an extremely small time interval. Hence for the interval, the truncated Taylor expansion is applied with high precision:

$$
\begin{aligned}
& \exp (\mathrm{H} \tau) \approx \mathrm{I}_{\mathrm{n}}+\mathrm{H} \tau+\frac{(\mathrm{H} \tau)^{2}}{2 !}+\frac{(\mathrm{H} \tau)^{3}}{3 !}+\frac{(\mathrm{H} \tau)^{4}}{4 !} \\
& \mathrm{T}_{\mathrm{a}}=\mathrm{H} \tau+\frac{(\mathrm{H} \tau)^{2}}{2 !}+\frac{(\mathrm{H} \tau)^{3}}{3 !}+\frac{(\mathrm{H} \tau)^{4}}{4 !}, \exp (\mathrm{H} \tau) \approx \mathrm{I}_{\mathrm{n}}+\mathrm{T}_{\mathrm{a}} \\
& \mathrm{T}=\left(\mathrm{I}_{\mathrm{n}}+\mathrm{T}_{\mathrm{a}}\right)^{2^{N}}=\left(\mathrm{I}_{\mathrm{n}}+\mathrm{T}_{\mathrm{a}}\right)^{2^{(N-1)}} \times\left(\mathrm{I}_{\mathrm{n}}+\mathrm{T}_{\mathrm{a}}\right)^{2^{(N-1)}}
\end{aligned}
$$

The last four term series expansion $T_{a}$ is extremely small from the unit matrix $\mathrm{I}_{\mathrm{n}}$ and will become an appended part, so its precision will be seriously dropped in the round-off operation in computer arithmetic if the multiplication was executed after the addition of the unit matrix. From the identity

$$
\left(\mathrm{I}+\mathrm{T}_{\mathrm{a}}\right) \times\left(\mathrm{I}+\mathrm{T}_{\mathrm{a}}\right)=\mathrm{I}+\left(\mathrm{T}_{\mathrm{a}}+\mathrm{T}_{\mathrm{a}}+\mathrm{T}_{\mathrm{a}} \times \mathrm{T}_{\mathrm{a}}\right)
$$

We can calculate the $\mathrm{N}$ times multiplication of $\mathrm{T}_{\mathrm{a}}$ corresponded to the following instruction:

$$
\text { for }(\text { iter }=0 \text {; iter }<\mathrm{N} ; \text { iter }++) \mathrm{T}_{\mathrm{a}}=2 \mathrm{~T}_{\mathrm{a}}+\mathrm{T}_{\mathrm{a}} \times \mathrm{T}_{\mathrm{a}}
$$

After the execution of the above instruction, $T_{a}$ is no longer a very small matrix, and this addition will have no serious numerical round-off error again. The exponential matrix $\mathrm{T}$ is achieved finally as bellow:

$$
\mathrm{T}=\left(\mathrm{I}+\mathrm{T}_{\mathrm{a}}\right)
$$

Then the state variable vector at any time step $\mathrm{V}_{1}, \mathrm{~V}_{2}, \ldots, \mathrm{V}_{\mathrm{k}}, \ldots$ is obtained. The algorithm given above is called the precise computation of exponential matrix.

\subsection{Krylov subspace based projection method}

Based on modern control theory, the dynamic systems (1) could be written in the state space form:

$$
\left\{\begin{array}{c}
\dot{\mathrm{v}}=\mathrm{A} \cdot \mathrm{v}+\mathrm{B} \cdot \mathrm{u} \\
\mathrm{y}=\mathrm{C} \cdot \mathrm{v}
\end{array}\right.
$$


where $\mathrm{v} \in \mathrm{R}^{\mathrm{n}}$ is the state variable, $\mathrm{A} \in \mathrm{R}^{\mathrm{n} \times \mathrm{n}}$ is system matrix function, $\mathrm{B} \in \mathrm{R}^{\mathrm{n} \times \mathrm{m}}$ is input matrix, $C \in R^{p \times n}$ is output matrix, $u \in R^{m}$ is input variable, $y \in R^{p}$ is output variable. Its frequency domain transfer function $\mathrm{G}(\mathrm{s})$ as

$$
\mathrm{G}(\mathrm{s})=\mathrm{C} \times(\mathrm{sI}-\mathrm{A})^{-1} \times \mathrm{B}=-\mathrm{C} \times \mathrm{A}^{-1}\left(\mathrm{I}-\mathrm{sA}^{-1}\right) \mathrm{B}
$$

$\mathrm{G}(\mathrm{s})$ can expand in Taylor series about some point, e.g. $\mathrm{s}=0$, so get polynomial format

$$
\mathrm{G}(\mathrm{s})=-\mathrm{CA}^{-1}\left(\mathrm{I}+\mathrm{sA}^{-1}+\mathrm{s}^{2} \mathrm{~A}^{-2}+\ldots\right) \mathrm{B}=-\sum_{\mathrm{k}=0}^{\infty} \mathrm{m}_{\mathrm{k}} \mathrm{s}^{\mathrm{k}}
$$

the kth coefficient $m_{k}=C^{-k}\left(A^{-1} B\right)$ is called the $k$ th moment of the transfer function. A r-dimension Krylov subspace is defined as follows

$$
\kappa_{\mathrm{r}}(\mathrm{A}, \mathrm{b})=\operatorname{span}\left\{\mathrm{b}, \mathrm{Ab}, \mathrm{A}^{2} \mathrm{~b}, \ldots, \mathrm{A}^{\mathrm{r}-1} \mathrm{~b}\right\}
$$

where $\mathrm{A} \in \mathrm{R}^{\mathrm{n} \times \mathrm{n}}, \mathrm{b} \in \mathrm{R}^{\mathrm{n}}$. The Arnoldi process [5] can create the orthonormal vectors to construct the unit basic $\mathrm{W}_{\mathrm{r}}=\left[\mathrm{w}_{1}, \mathrm{w}_{2}, \ldots \mathrm{W}_{\mathrm{r}}\right]$ for Krylov subspace, $\mathrm{W}_{\mathrm{r}} \in \mathrm{R}^{\mathrm{n} \times \mathrm{r}}$. If original system (15) was projected to the $\mathrm{r}$-dimension Krlov subspace, $\mathrm{r} \ll \mathrm{n}$, get

$$
\left\{\begin{array}{c}
\dot{\hat{\mathrm{v}}}=\hat{\mathrm{A}} \cdot \hat{\mathrm{v}}+\hat{\mathrm{B}} \cdot \mathrm{u} \\
\mathrm{y}=\hat{\mathrm{C}} \cdot \hat{\mathrm{v}}
\end{array}\right.
$$

where $\hat{A}=W_{r}{ }^{T} \cdot A \cdot W_{r}, \hat{B}=W_{r}{ }^{T} \cdot B, \hat{C}=C \cdot W_{r}, \hat{v} \in R^{r}, \hat{G}(s)=\hat{C} \cdot(s I-\hat{A})^{-1} \cdot \hat{B}$

The previous research [6] have indicated that the first rth moments between $G(s)$ and $\hat{G}(s)$ are matching. So the output dynamic characteristic of original system (15) could be described by reduced order systems (19).

\subsection{MOR-PIM method concept}

The flow diagram of MOR-PIM method for solving large-scale nonlinear dynamics system is shown in Fig 1. The simulation process consists of four basic steps as follows:

(a).Decompose nonlinear matrix functions to time-invariant part and external inhomogeneous term. The time-invariant matrix obtained here will be convenient for model order reduction and time integrating in the last two steps process.

(b).Transform the non-homogeneous equations into homogenous ones with dimensional expanding process. It can avoid inverse matrix calculations and storage.

(c). Reduce the increment-dimensional systems order through all kinds of model order reduction method as you can. Krylov subspace based projection method has advantage of standardization and automation. It has efficient effect for linear timeinvariant system. Some other improved method, such as SVD-Krylov [7], Padé via Lanczos [8], Rational Krylov method [9] would also used to nonlinear systems.

(d).Take precise integration method for reduced order systems. A rapid transient analysis result for complicated coupled systems and the system-level simulation would be obtained efficiently and accurately. 


\section{Nonlinear Transient Analysis for Large-Scale Dynamics of Microelectromechanical Systems}

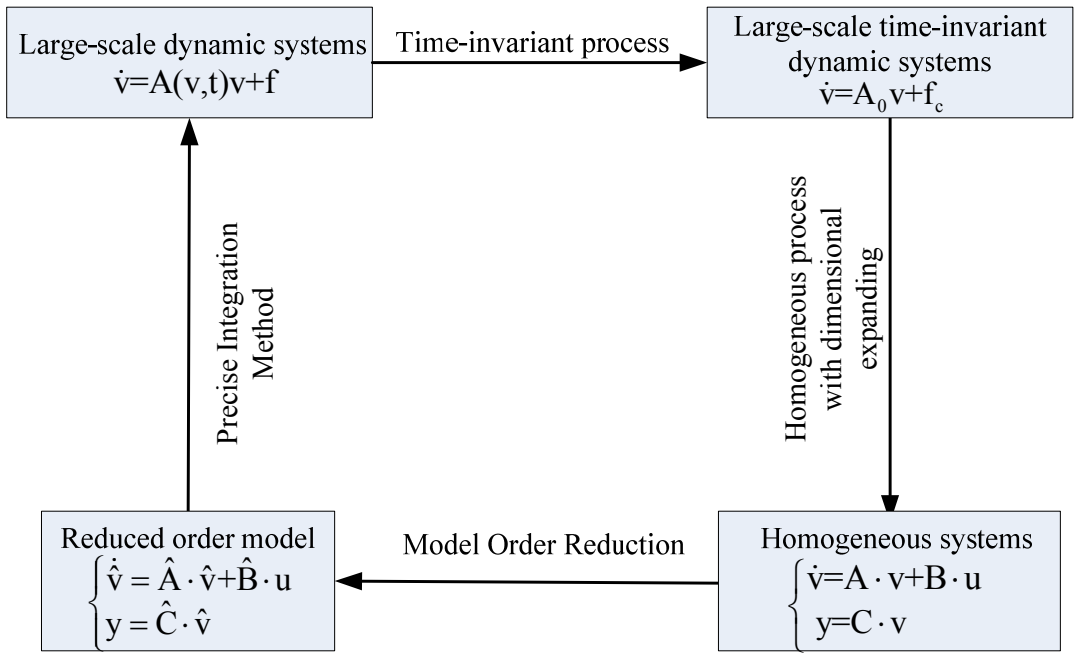

Fig.1 The flow diagram of MOR-PIM method for large-scale dynamic systems

\section{NUMERICAL EXAMPLES}

The polysilicon V-beam electro-thermal actuator is shown in Fig 2, after applying input current to the electrode, the joule heat will be generated in the actuator and the thermal expansion of structure would take the vertical displacement and actuate the microgripper to achieve clamping action.

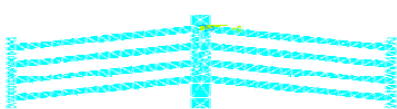

(a)

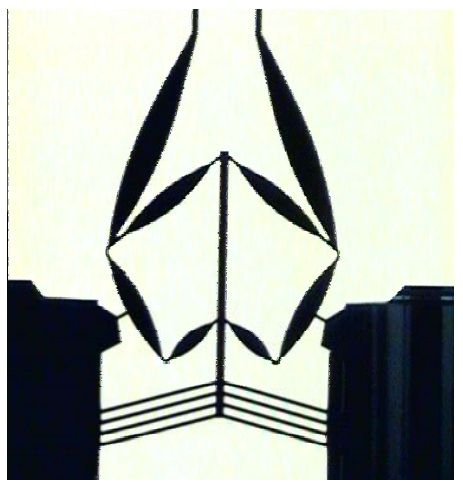

(b)

Fig.2 (a) The finite element model of V-beam electro-thermal actuator

(b) The micrograph of $\mathrm{V}$-beam electro-thermal actuator and microgripper

The finite element model of V-beam actuator was built in Ansys. Ansys performs the electro-thermal-structural analysis using sequential coupling, where the system functions was assembled just like in [10] using Mor4ansys [11]. The nodes number of this finite element model is 11722 . The transient analysis result using ANSYS and different number reduced order model by MOR-PIM method using Matlab is shown in Fig 3. The result shows that the concept of MOR-PIM method is effective and it is saving computing time and memory storage than commercial FEM software. 


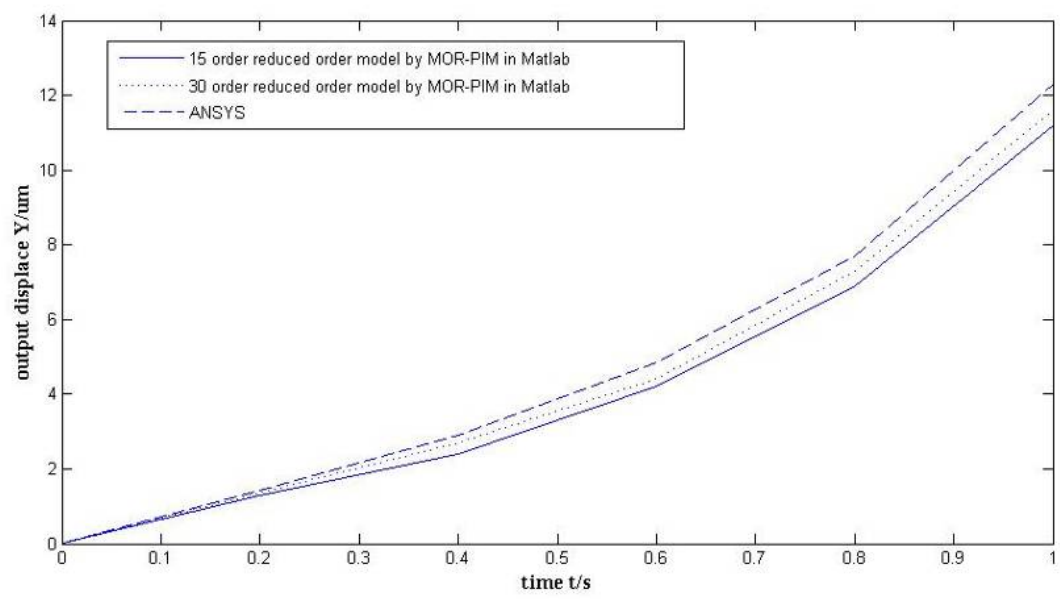

Fig.3 Transient analysis with ANSYS, 30 and 15 order reduced model using MOR-PIM

\section{CONCLUSION}

An MOR-PIM technique is present to solve large-scale nonlinear finite element dynamics systems. Firstly the state variables depended with time or related to unknown vector can be treated as external force vectors just like inhomogeneous terms; then the inhomogeneous equation is converted into homogeneous equation through incrementdimensional method by which the non-homogeneous vectors is viewed as the state variables of equations; thirdly the Krylov subspace projection method is used to reduce model order for large-scale homogeneous dynamics systems. Finally the rapid transient response of reduced order model is solved by precise integration method. This method takes advantage of the excellence between model order reduction and precise integration method, which is worth to be improved and developed in future.

\section{ACKNOWLEDGEMENT}

This research is supported by the National Basic Research Program of China (Grant No.2011CB302101, 2011CB302105) and the Fundamental Research Funds for the Central Universities (Grant No. DUT10ZD104).

\section{REFERENCES}

1. A. H. Nayfeh, M. I. Younis, and E. M. Abdel-Rahman, Reduced-order models for MEMS applications, Nonlinear Dynamics 41, 211-236, 2005

2. K. Subbara and M. A. Dokainish, A survey of direct time integration methods in computational structural dynamics: I Explicit methods, II Implicit methods, Computers \& Structures 32, 371-1386, 1387-1401, 1989.

3. W. X. Zhong and F. W. Williams, A precise time step integration method, Journal of Mechanical Engineering Science 208, 427-430, 1994. 
4. Y. X. Gu, B. S. Chen, H. W. Zhang and Z. Q. Guan, Precise time-integration method with dimensional expanding for structural dynamic equations, AIAA Journal 39, 2394-2399, 2001.

5. W. E. Arnoldi, The principle of minimized iterations in the solution of the matrix eigenvalue problem, Quart Appl Math 9, 17-29, 1951.

6. E. J. Grimme, Krylov projection methods for model reduction, University of Illinois at Urbana Champaig, 1997.

7. S. Gugercin, An iterative SVD-Krylov based method for model reduction of largescale dynamical systems, Proceedings of the 44th IEEE Conference on Decision and Control, 5905-5910, 2005.

8. P. Feldmann and R. Freund, Efficient linear circuit analysis by Padé approximation via the lanczos process, IEEE Trans. Computer-Aided Design, 639-649, 1995.

9. S. Gugercin, Projection methods for model reduction of large-scale dynamical systems, Rice University, Houston, 2003.

10. J. K. Chu, L. Guan, D. Z. Qi, and X. C. Hao, Rapid Nonlinear Analysis for Electrothermal Microgripper Using Reduced Order Model Based on Krylov Subspace, International Journal of Nonlinear Sciences and Numerical Simulation 9, 333-338, 2008.

11. E. B. Rudnyi, J. Lienemann, A. Greiner and J. G. Korvink, Mor4ansys: Generating Compact Models Directly from ANSYS Models, Technical Proceedings of the 2004 Nanotechnology Conference and Trade Show, Nanotech, 2004. 